\title{
POUR UNE SOCIOLOGIE DE LA SOCIÉTÉ TRANSNATIONALE
}

\author{
Abram DE SWAAN
}

Résumé : Afin de proposer un cadre général pour l'étude de la société transnationale actuellement en émergence, l'article examine de manière critique les principales démarches disciplinaires en cours aujourd'hui. Il souligne l'intérêt d'une approche fondée sur un modèle de type centre/périphérie accompagné de l'hypothèse d'une compétition oligopolistique. Avec ce cadre d'analyse général, la sociologie peut identifier son objet dans le système des liens établis entre les êtres humains par-delà les frontières nationales, que ces liens soient directs ou médiatisés par des institutions transnationales. Une version plus élaborée, et révisée, d'un tel modèle est notamment illustrée par la dynamique de la globalisation culturelle, et par le «système mondial des langues ».

Mots-Clés : culture globale, société mondiale, culture mondiale, centre/périphérie, réseaux, modèle.

ABSTRACT : Proposing a general framework for the study of the emerging transnational society, the article critically surveys predominant disciplinary perspectives in this field, and outlines an approach based on a core/periphery model with oligopolistic competition. Within this general framework, sociology finds its specific subject in the ties that connect human beings across national borders, directly or mediated by transnational institutions. An elaborated and amended version of this approach is illustrated, among others, by the dynamics of cultural globalization and the "world language system".

KEYWORDs : global culture, global society, world culture, core/periphery, networks, pattern. Revue de synthèse : $4^{\mathrm{e}} \mathrm{S} . \mathrm{n}^{\mathrm{o}} 1$, janv.-mars 1998, p. 89-111. 
ZUSAMMENFASSUNG: In der Absicht, einen allgemeinen Rahmen für die Untersuchung der entstehenden transnationalen Gesellschaft vorzuschlagen, wird in diesem Artikel eine Kritische Darstellung der wichtigsten aktuellen Perspektiven in diesem Gebiet gegeben. Dabei wird die Bedeutung eines Ansatzes hervorgehoben, der mit einem Modell vom Typ Zentrum/Peripherie arbeitet, unter der Annahme eines oligopolistischen Wettbewerbs. Innerhalb dieses allgemeinen Rahmens kann die Soziologie ihren speziellen Gegenstand in den Bindungen finden, die zwischen einzelnen Menschen über nationale Grenzen hinweg bestehen. Diese Bindungen können direkt sein oder durch transnationale Organisationen bedingt werden. Eine ausgearbeitete und verbesserte Version eines solchen Modells wird unter anderem am Beispiel der Dynamik der kulturellen Globalisierung und durch das "Weltsystem der Sprachen " verdeutlicht.

STICHWORTER : Globalisierung der Kultur, Weltgesellschaft, Weltkultur, Zentrum/Peripherie, Vernetzung, Modell.

Sommarıo : Al fine di offrire un quadro generale per lo studio della società transnazionale attualmente emergente, l'articolo esamina in modo critico le principali procedure disciplinari oggi in corso. Si sottolinea l'interesse di un approccio fondato su un modello del tipo centro/periferia, insieme all'ipotesi di una competizione oligopolistica. Grazie a questo quadro generale, la sociologia può identificare il proprio oggetto nel sistema dei rapporti fra gli esseri umani al di là delle frontiere nazionali, siano essi legami diretti o rapporti mediati da istituzioni transnazionali. Una versione più elaborata di questo modello è illustrata proprio dalla dinamica della globalizzazione culturale e dal " sistema mondiale delle lingue ".

Parole ChIAvE : cultura globale, società mondiale, cultara mondiale, centro/periferia, rete, modello.

Abram DE SwaAN, né en 1942, occupe la chaire de sociologie à l'université d'Amsterdam depuis 1973. Il est président de l'école d'Amsterdam de recherches en sciences sociales (ASSR). Il a publié en 1988 un livre sur la sociogenèse de l'État-providence, traduit en français en 1995, Sous l'aile protectrice de l'État (Paris, Presses universitaires de France). Pendant l'année 1997/1998, il est titulaire de la chaire européenne du Collège de France «Langue et culture dans la société transnationale ".

Adresse : De Amsterdamse School voor Sociaalwetenschappelijk Onderzoek, Oude Hoogstraat 24, 1012 CE Amsterdam, Pays-Bas.

Courrier électronique : a.deswaan@mail.uva.nl 
«Die Kreise der sozialen Interessen liegen konzentrisch um uns. »

Georg Simmel, Über soziale Differenzierung, Leipzig, Duncker \& Humblot, 1890, p. 3.

pour Johan GoudsBLom

Les sociologues manient le concept de "société » comme l'unité d'analyse la plus étendue, comme un concept qui, aux yeux de ces enfants du siècle, coïncide avec l'État-nation. Mais les fondateurs des sciences sociales envisageaient cela sous un horizon plus vaste. Ainsi, pour Adam Smith, la "richesse des nations » résultait de la division internationale du travail, et Karl Marx voyait le capitalisme autant comme le produit d'un réseau planétaire de relations d'échanges que comme la force qui lui fournit son impulsion!.

C'est seulement après que les sciences sociales furent assimilées par l'enseignement universitaire sous le patronage de l'État que la perspective nationale s'imposa. Dans la division du travail universitaire dominante, les recherches sur un sujet outrepassant le cadre national relevèrent alors de spécialités telles que l'économie internationale, les relations internationales ou encore le droit international. Les organisations internationales apparurent finalement comme de nouvelles venues parmi les matières enseignées. Quant aux sociétés encore épargnées par le modèle de l'État-nation, elles tombèrent sous l'empire de l'anthropologie.

C'est seulement depuis une date récente que les sociologues se préoccupent à nouveau d'évolutions sociales qui déborderaient un cadre purement national. Les travaux d'Immanuel Wallerstein sur les origines du système mondial moderne, qui conjuguent économie, histoire et analyse sociale, sont pour beaucoup dans ce regain d'intérêt. Son autre ressort est la renaissance de l'histoire mondiale telle qu'elle est pratiquée par William McNeil, Alfred W. Crosby, Philip Curtin, Eric L. Jones et, aux Pays-Bas même, par Johan Goudsblom².

1. Voir Karl MarX, Das Kapital. Kritik der politischen Ökonomie, 1867, t. I, part. VII, chap. xxıv, \& 7, Berlin, Dietz, 1973, p. 790 : « [...] die Verschlingung aller Völker in das Netz des Weltmarkts und damit der internationale Charakter des kapitalistischen Regimes. "

2. Le débat entre ces auteurs a lieu dans le Journal of world history, revue qui paraît depuis 1989. 
Adoptant une perspective sociologique, d'autres chercheurs ont abordé la question de la «société mondiale » actuelle : dès 1972, John W. Burton plaida en faveur d'une étude des rapports transnationaux qui se nouent entre les personnes, et pas seulement sous l'égide des seules relations entre les États :

«Si l'on superposait des transparents figurant les déplacements hebdomadaires effectués en avion par les passagers, les flots de télégrammes, les relations de nature ethnique ou linguistique, les voyages effectués par les savants et les conseillers techniques, les mouvements migratoires, les touristes et tous les autres types de transactions, on pourrait se faire une idée de ces relations qui nous mettrait mieux à même d'expliquer le comportement des hommes dans la société mondiale que nous ne le pouvons partant des cartes traditionnelles ${ }^{3}$. »

Vingt ans plus tard, mais dans le même esprit, Leslie Sklair défend le principe d'une «sociologie du système mondial » qui devrait s'intéresser aux pratiques transnationales ${ }^{4}$. Optant pour une perspective néo-marxiste, cet auteur se préoccupe surtout des relations économiques:

«Le système mondial se compose de pratiques économiques transnationales. Celles-ci forment au niveau d'abstraction le plus élevé les éléments constitutifs du système. Les pratiques politiques en sont les principes d'organisation [...] Les pratiques idéologico-culturelles sont les boulons et les écrous qui assurent la cohésion du système ${ }^{5}$. »

Les travaux d'Evan Luard proposent une autre approche sociologique :

«Il vaudrait mieux concevoir la recherche portant sur la société internationale, y compris sur les relations entre États comme portant sur la société en général : soit à proprement parler comme relevant de la sociologie ${ }^{6}$.»

Cependant, après l'examen d'une série de concepts sociologiques fondamentaux, tels «action », « rôle » ou « autorité », Luard les applique à la société internationale sans réussir à ramener cette vie sociale transnationale à une conceptualisation sociologique synthétique.

C'est sous la rubrique global culture («culture mondiale » ou «culture globale ») qu'on range aujourd'hui bon nombre d'exposés traitant de la

3. John W. Burton, World Society, Cambridge, Cambridge University Press, 1973, p. 35.

4. Leslie SkLaIR, Sociology of the global system, Baltimore, Johns Hopkins University Press, 1991, p. 5-6 (en italiques dans l'original).

5. Ibid., p. $81-82$.

6. Evan LuARD, International Society, Londres, Macmillan, 1990, p. vII. On trouvera dans cet ouvrage une bonne synthèse des résultats d'un certain nombre de monographies antérieures sur la société internationale. 
société transnationale, un genre très au goût du jour. Bien que Janet AbuLughod en ait, de manière probante, caractérisé la plus grande part comme du « global babble ${ }^{7}$ », on ne trouve pas moins sous cette étiquette, précisément, quelques-uns des textes anthropologiques et sociologiques récents les plus intéressants ${ }^{8}$. Ils ont pour thème principal la dialectique entre le «mondial » et le «local» («the glocal» a pu dire Robert Robertson), ou encore celle entre des vocabulaires universels ou particuliers. Assiste-t-on, en effet, à la naissance d'une culture uniforme et planétaire, d'une culture unique? Et si c'est le cas, est-elle synonyme d'occidentalisation et même d'américanisation (c'est la thèse de George Ritzer ${ }^{9}$ ) ou bien cette culture procède-t-elle d'échanges à double sens entre centre et périphérie selon un processus d' « indigénisation » et de "créolisation » (c'est celle d'Ulf Hannerz $\left.{ }^{10}\right)$ ? Ou bien encore, revenant à la première question, existe-t-il au contraire une diversité de cultures transnationales qui ont leur origine non seulement en Europe et aux États-Unis mais également en Afrique, en Asie ou encore en Amérique latine (voyez cette fois Arjan Appadurai ${ }^{11}$ )?

Or il s'avère difficile aujourd'hui de distinguer entre critique et analyse de la culture mondiale. L'École de Francfort a donné le ton en animant la polémique visant la «culture populaire ", la «culture de masse », en particulier dans ses manifestations d'origine américaine, laissant ininterrogée la culture dominante dans son acception européenne. Ce sont des historiens tels Lawrence Levine ${ }^{12}$ qui ont repéré l'origine sociale de la séparation

7. Voir Janet ABU-Lughod, « Going beyond global babble ", in Culture, globalization and the World-system. Contemporary conditions for the representation of identity, éd. Anthony D. KING, Binghamton. NY/Londres, State University of New York at Binghamton/Macmillan, 1991 , p. 131-138.

8. Voir notamment : Globalization, knowledge and society, éd. Martin Albrow, Elizabeth KING, Londres, Sage, 1990; Global Culture. Nationalism, globalization and modernity, éd. Michael Feather-Stone, Londres, Sage, 1991 (également un numéro spécial de Theory, culture and society, 1990); Ulf HANNERZ, Cultural Complexity. Studies in the social organization of meaning, New York, Columbia University Press, 1992; op. cit. supra n. 7; George RITZER, The McDonaldization of society. An investigation into the changing character of contemporary social life, Londres/Thousand Oaks, Sage/Fine Forge Press, 1993; Robert Robertson, Globalization. Social theory and global culture, Londres, Sage, 1992; Immanuel Wallerstein, Geopolitics and geoculture. Essays on the changing world-system, Cambridge/ Paris, Cambridge University Press/Maison des sciences de l'homme, 199i.

9. G. Ritzer, op. cit. supra n. 8.

10. L'autre face du diktat colonial et post-colonial de l'authenticité, c'était l'idée que la diffusion de la culture de masse équivalait à l'annihilation des formes culturelles indigènes. En réalité, il se passait et il se passe autre chose : la promiscuité cosmopolite, ou, pour utiliser la métaphore plus appropriée d'U. Hannerz, « la créolisation ». Voir U. HANNERZ, "The world in creolisation », Africa, vol. 57, 1987, p. 546-559 et ID., op. cit. supra n. 8, p. 261-267.

11. Arjan Appadurai, «Disjuncture and difference in the global cultural economy », in Global Culture, op. cit. supra n. 8, p. 295-310.

12. Lawrence W. LEvinE, Highbrow Lowbrow. The emergence of cultural hierarchy in America, Cambridge, MA, Harvard University Press, 1988; voir, également, Cultivating Differences. Symbolic boundaries and the making of inequality, éd. Michèle LamonT, Marcel Fournier, Chicago, University of Chicago Press, 1992. 
entre « haute » ( « high») culture et culture « populaire» («low»), et des sociologues comme Herbert Gans ${ }^{13}$, des chercheurs de l'École de Birmingham $^{14}$, et bien entendu Pierre Bourdieu ${ }^{15}$, qui ont décrit les fonctions sociales de cette distinction. Dans l'analyse de la culture mondiale d'aujourd'hui, l'opposition entre l'«authenticité » de la culture «traditionnelle » ou « indigène » et la «culture-de-masse-commerciale-del'Ouest " remplit une fonction analogue à l'ancienne opposition entre « haute " culture et culture « populaire », en sorte qu'il s'avère tout aussi difficile qu'hier d'objectiver cette nouvelle distinction.

Ce sont des anthropologues, enquêteurs des pratiques culturelles spécifiques in situ, qui nous fournissent quelques-unes des remarques les plus lucides sur la question. Ils ont en effet observé de près les transformations qui affectent les usages et la signification d'éléments culturels une fois transplanté du contexte propre à une société vers un autre ${ }^{16}$. Aucun produit, et pas même les articles de consommation «de masse » et les séries télévisées retransmises par satellites, n'est consommé passivement, ni sans nouvelles intermédiations. Recevant ces produits, les consommateurs les soumettent à de nouvelles interprétations et applications à leur propre cadre de vie.

Dans un schéma transnational, de telles pratiques culturelles se trouvent insérées dans des réseaux de relations d'échanges économiques et d'interdépendance politiques riches en controverses, en tensions et en provocations, riches encore en reprises conformistes ou subversives, ces réseaux rayonnant des zones centrales en direction des zones périphériques. Aussi arrive-t-il que des versions modifiées retournent au centre pour être à nouveau re-distribuées à l'échelle planétaire dans ce nouvel état : la musique, la world music, est l'un des exemples le plus parlant d'une telle créolisation ${ }^{17}$.

On crée, on distribue, on transforme et on consomme la culture mondiale, ou la culture globale comme on voudra, dans des contextes sociaux spécifiques. Cette culture est, par conséquent, nécessairement insérée dans des réseaux spécifiques qui relient entre eux les gens, et ce nécessairement

13. Herbert J. Gans, Popular Culture and high culture. An analysis and evaluation of taste, New York, Basic Books, 1974 ; voir, également, ID., «Introduction ", in Cultivating Differences, op. cit. supra n. 12, p. vil-Xvi.

14. Par ex., Culture, media, language, éd. Stuart HaLL, Dorothy HoBson, Andrew Lowe, Paul Willis, Londres, Hutchinson, 1984; Dick Hebdige, Subculture, Londres, Methuen, 1979.

15. Pierre Bourdieu, La Distinction, Paris, Minuit, 1979.

16. Par ex., Justin-Daniel Gandoulou, Entre Paris et Bacongo, Paris, centre Georges Pompidou, 1984.

17. Voir Abram de SwaAN, «Alles is in beginsel overal (maar de Mosselman is nergens meer). Over het internationaal cultuurstelsel en het nationaal cultuurbeleid », in ID., Perron Nederland, Amsterdam, Meulenhoff, 1991, p. 93-120. 
par-delà les frontières nationales. En d'autres termes, on ne peut envisager séparément l'étude de la culture globale et celle de la société mondiale.

DES LIENS QUI DÉPASSENT LES FRONTIÈRES

La sociologie de la société transnationale a ainsi pour tâche première d'étudier les relations transnationales : celles qui lient les personnes entre elles par-delà les frontières, directement ou par l'entremise des gouvernements, d'entreprises transnationales et d'autres organisations. C'est pourquoi cette sociologie doit moins s'attacher à rendre compte des relations entre États que des rapports existant entre les ressortissants des différents pays, et, dès lors, au rôle que jouent les États, les entreprises et les institutions internationales dans l'établissement de ces rapports. Voici, par exemple, deux contributions qui illustrent au mieux cette approche : Longdistance Nationalism de Benedict Anderson ${ }^{18}$ et Cultural Complexity d'Ulf Hannerz ${ }^{19}$ - deux anthropologues soit dit en passant.

La sociologie de la société transnationale doit encore savoir s'affranchir des thèmes qui relevaient jusqu'ici de l'économie internationale. Elle a mieux à faire en effet en étudiant, par exemple, la façon dont les liens s'établissent, par la médiation des entreprises, entre les individus d'une part - au titre d'employés, de consommateurs ou encore de propriétaires et d'actionnaires - et les marchés internationaux d'autre part.

En troisième lieu, la sociologie de la société transnationale ne doit pas se contenter d'analyser la culture mondiale comme un phénomène autonome. Il lui faut au contraire étudier le réseau des relations entre les différents genres de publics et les différents types de consommateurs tel qu'il est apparu parallèlement au surgissement des médias de masse planétaires et des canaux mondiaux de distribution.

En étudiant les liaisons qui existent entre les personnes par-delà les frontières, il devient ainsi possible de dépasser les limites des objets traditionnels des disciplines universitaires et de délimiter un domaine de recherche relativement autonome ayant sa place à côté de disciplines comme les relations internationales et l'économie internationale. Sa particularité est de rendre possible l'interprétation de phénomènes culturels à partir des différentes échelles qui les caractérisent, mondiale, nationale et locale.

18. Benedict R. O. Anderson, Long-distance Nationalism. World capitalism and the rise of identity politics, Amsterdam, Centre for Asian Studies Amsterdam, 1992.

19. U. HANNERZ, op. cit. supra n. 8. 
DENSIFICATION ET EXTENSION DU RÉSEAU TRANSNATIONAL

Au cours des cinquante dernières années, le réseau des relations transnationales a subi de véritables bouleversements. Devenu à la fois plus intensif et plus extensif, plus diversifié et plus complexe. De telles transformations ne sont pas toutefois sans précédents, parfois assez lointains dans le temps.

À plusieurs égards, les élites artistiques, universitaires et aristocratiques de l'Europe des $\mathrm{XVII}^{\mathrm{e}}$ et $\mathrm{XVIII}^{\mathrm{c}}$ siècles se trouvèrent moins confinées dans les limites d'un territoire donné que celles du $\mathrm{XIX}^{\mathrm{e}}$ et du début du $\mathrm{xx} \mathrm{x}^{\mathrm{e}}$ siècle. Leur horizon était plus «continental ». À partir du bas Moyen Âge, les artistes parcoururent l'Europe, allant d'une cour à une autre. Les étudiants faisaient pour leur part la tournée des villes universitaires. Il n'était pas rare de voir les savants passer le plus clair de leur temps à l'étranger, soit dans des couvents soit dans des universités ou encore auprès d'un seigneur étranger. De même, les souverains prémodernes et ceux de l'aube de l'ère moderne étaient-ils comme apatrides. Ces princes trouvaient leurs épouses au-delà des frontières de leurs possessions, s'exprimaient dans une langue étrangère et s'établissaient souvent là où un trône venait à être vacant. Sur le continent, les lingua franca se succédèrent - le latin, l'italien puis le français. Elles facilitaient les échanges entre lettrés de toutes origines et de toutes langues ${ }^{20}$.

Mais cet « internationalisme » antérieur aux nations, ou plutôt pour être plus rigoureux ce « continentalisme prénational » (limité principalement au sous-continent européen), suivant une tradition remontant au haut Moyen Âge et même à une époque plus ancienne, était uniquement l'apanage d'une couche supérieure très réduite du clergé, de la noblesse et du monde artistique et scientifique.

De nos jours, l'internationalisation est comme une contrepartie de la formation des nations : elle accompagne la formation des États nationaux et s'y oppose dans le même temps. Depuis disons 1815 (le congrès de Vienne), on a progressivement divisé la surface habitable du globe en États nationaux dont les frontières, en plus de leur signification géographique, ont fonctionné comme des barrières entre les différentes cultures nationales qui se formaient sur chaque territoire. Par contrecoup, les élites culturelles et intellectuelles du $\mathrm{XIX}^{c}$ siècle eurent une tendance croissante à se satisfaire de leur propre cadre national. Parallèlement, toutefois, on assistait à une expansion toujours plus rapide du capitalisme industriel, les marchés venant à franchir toujours plus avant les frontières étatiques.

20. Voir Claude Hagég, Le Souffle de la langue. Voies et destins des parlers d'Europe, Paris, Odile Jacob, 1992. 
Le colonialisme occasionna lui aussi un processus de transgression des frontières; opérant loin de l'Europe, plusieurs États se construisirent un empire assis sur un pouvoir fragile mais géographiquement étendu, et leur domination extensive contrastait avec leur manière intensive de gouverner et d'administrer en métropole même. Après la Seconde Guerre mondiale, ces puissances coloniales durent faire face aux formes d'opposition manifestée par la population indigène. Elles eurent à renoncer aux formes les plus intensives de domination et à mettre sur pied des relations économiques de substitution; elles abandonnèrent ainsi la mainmise politique et administrative aux nouvelles élites locales post-coloniales et nationalistes.

Certes, le monde antique a connu des empires aussi vastes que ceux des $\mathrm{XI}^{\mathrm{e}}$ et $\mathrm{Xx}^{\mathrm{e}}$ siècles. Toutefois, il convient ici de distinguer les modalités de l'exercice du pouvoir impérial. On peut, en effet, qualifier de tendanciellement extensive la forme de domination ancienne, lourde mais en fait superficielle, par opposition à la forme actuelle, plutôt intensive qui se manifeste de manière emblématique dans la systématisation des appareils d'extraction fiscale des revenus ou encore par l'imposition de normes linguistiques. L'intensification aujourd'hui réalisée a été rendue possible par la croissance exponentielle des moyens de transport et de communication en même temps que par l'accroissement de la productivité des techniques militaires et administratives.

Depuis quelques années, on assiste de surcrô̂t à un processus de décomposition de l'ex-Union soviétique, situation confuse d'où surgissent de nouvelles entités nationales. Là où ils se forment, on observe des États renforcer sur leur territoire la domination qu'ils exercent au rythme même où ils deviennent plus dépendants d'autres États : ces pays connaissent en même temps un processus de formation nationale et un processus d'insertion internationale, disons d'internationalisation. De plus en plus, les États nationaux dépendent dans leur formation du système étatique mondial, lequel leur impose ses normes et leur procure une reconnaissance internationale et renforce du même coup leur légitimité à l'intérieur même de leurs frontières ${ }^{21}$.

DIFFUSION DES ORIENTATIONS INTERNATIONALES

Ce sont les systèmes d'enseignement - en particulier l'enseignement secondaire en ce qu'il impose l'apprentissage des langues étrangères - qui

21. Au sujet de la façon dont les États post-coloniaux ont adopté les institutions politiques de l'ancienne puissance coloniale, voir Bertrand BADIE, L'État importé. Essai sur l'occidentalisation de l'ordre politique, Paris, Fayard, 1992; Basil Davidson, The Black Man's burden. Africa and the curse of the Nation State, Londres, James Curry, 1993. 
est l'un des principaux facteurs de la formation de la société transnationale. Le pourcentage d'élèves inscrits dans des établissements secondaires a connu une augmentation spectaculaire au cours des cinquante dernières années $^{22}$. Le moteur que fut l'enseignement primaire pour le processus de formation des nations trouve de nos jours son pendant pour ce qui est du processus de mondialisation dans l'augmentation du nombre des élèves des collèges et des lycées qui assurent des enseignements des grandes langues véhiculaires ainsi que des codes mondiaux que sont les mathématiques et les sciences naturelles.

Les médias électroniques représentent un autre nerf de l'internationalisation des masses en produisant et en diffusant une culture populaire qui ne connaît pratiquement plus de frontière aujourd'hui. Ce qui est vrai pour les divertissements commerciaux l'est a fortiori pour le phénomène comparable des sports de grande audience et la distribution au niveau planétaire d'articles de marque. Un élément de plus dans cette révolution de la communication est le renouvellement permanent qui affecte la représentation des autres peuples, c'est-à-dire de la catégorie «étrangers », tout particulièrement au moyen des reportages et des sujets d'information télévisés. On ne sait encore, à vrai dire, que peu de choses sur l'impact qu'exerce ce flot continu d'informations, systématiquement élaborées, sur le public qu'il touche. Mais selon toute vraisemblance, il n'est pas étranger au revirement qu'on a pu observer de la part des organisations transcontinentales de bienfaisance qui manifestent dorénavant plus d'activisme en la matière et mettent en avant la dramatisation et le côté visuel de la souffrance des malheureux comme de l'aide qu'elles leur apportent ${ }^{23}$. Parallèlement, la culture médiatique populaire et transnationale se répercute dans la nature des sentiments de solidarité suscités par des événements qui se déroulent par-delà les frontières nationales. C'est ce que Thomas Haskell a pu appeler «la sensibilité humanitaire ${ }^{24}$ ». Et même s'il s'agit d'un phénomène d'ampleur encore rela-

22. À propos de la diffusion mondiale des systèmes scolaires, voir John W. MEYER, Michael T. HANNAN, National Development and the world system. Educational, economic and political change, 1950-1970, Chicago, University of Chicago Press, 1979 et John W. MEYER, Francisco O. Ramirez, Yasemin Nuhoglu SoySAL, «World expansion of mass education, 1870-1980", Sociology of education, vol. 65, Apr. 1992, p. 128-149.

23. « Les organisations humanitaires occidentales ont dû, pour faire appel à la conscience de la population occidentale, dépeindre le Tiers monde comme une zone de catastrophes : tel est, hélas, l'un des effets secondaires involontaires de leur aide. Par conséquent, la plupart des gens se font du Tiers monde l'image stéréotypée d'un enfant africain qui meurt et qui tend la main pour avoir de la nourriture." Voir Peter WORSLEY, "Models of the modern worldsystem ", in Global Culture, op. cit. supra n. 8, p. 83-96, en part. p. 93. Voir, également, Luc Boltanski, La Souffrance à distance, Paris, Métailié, 1993.

24. Thomas L. HASKELL, "Capitalism and the origins of the humanitarian sensibility », I et II, American Historical Review, vol. 90, 2 et 3, 1985, p. 339-361 et 547-566. Voir également, Internationalism under strain. The North-South policies of Canada, the Netherlands, Norway, and Sweden, éd. Cranford PratT, Toronto, University of Toronto Press, 1989. 
tivement limitée, on observe de plus en plus de personnes s'identifier subjectivement à l'humanité considérée comme un tout planétaire ${ }^{25}$.

Partout où les médias de masse commerciaux sont de fait les supports de la culture populaire transnationale, on observe encore que «culture » et "économie » sont profondément intriqués. Les cultures nationales, dont chacune est très marquée par l'État dans lequel elle s'est déployée, se trouvent ainsi de plus en plus accompagnées, voire supplantées, par une culture transnationale qui se constitue principalement au gré des interactions des forces en présence sur le marché concurrentiel. C'est à la sociologie de la société transnationale qu'il revient de repérer les zones de tension entre ces deux sphères, entre les cultures nationales liées aux États dont elles relèvent et les cultures transnationales liées aux réseaux d'entrepreneurs opérant sur un marché planétaire.

\section{SYSTÈME DES ÉTATS ET SOCIÉTÉ TRANSNATIONALE}

Comment la société transnationale est-elle structurée? Qu'en dire au moins pour une première approche ? Cette société qui commence à se dessiner aujourd'hui se distingue de la société nationale pour trois raisons bien simples. En premier lieu, on y recense près de six milliards d'individus. Il est donc question d'un ordre de grandeur, strictement numérique mais aussi en terme d'hétérogénéité, comparable à aucune autre entité politique existante.

En deuxième lieu, on a affaire à une « société sans État» où ne s'est encore établi aucun monopole, et a fortiori aucun monopole efficace, de la violence légitime. Mais sans relever d'un système d'États, cette société englobe tous les États. C'est pourquoi les conflits y sont plus fréquents, plus étendus et plus difficiles à contrôler que dans les limites d'une entité étatique nationale. Cette société transnationale n'est pas pacifiée. Les conflits ne peuvent trouver de résolution que par les parties intéressées et par voie de concertation ou de confrontation violente. Il n'existe aucun appareil militaire disposant des moyens et du pouvoir de restaurer l'ordre sur la planète homologues des forces de police à l'intérieur des frontières d'un pays.

Troisièmement, enfin, force est de constater qu'à ce niveau planétaire, l'humanité se retrouve seule. Il n'existe aucune puissance extérieure pour la menacer ou la sauver, sauf à attendre la venue de quelque puissance stupéfiante qui relèverait pour le moins de la science-fiction.

25. Voir mon article, "Identificatie in uitdijende kring", Amsterdams Sociologisch Tijdschrift, jan. 1994, p. 6-24. 
Mais ces caractéristiques élémentaires ne sont pas sans antécédents. L'histoire de la formation des États présente, en effet, des signes avantcoureurs de la configuration mondiale que l'on connaît aujourd'hui, de tels États ayant il est vrai émergés à une échelle plus modeste pour ainsi dire avec et contre d'autres eux aussi en voie de formation. Au cours d'un tel prócessus, les seigneurs féodaux européens ne cessèrent de s'affronter, chacun s'efforçant de prendre le dessus sur l'adversaire et d'agrandir son territoire. Conquêtes et butins avaient pour conséquence le renforcement des troupes qui lui-même promettait de nouvelles expansions territoriales. Au cours d'un tel processus, les rivaux les moins puissants reconnaissaient leurs maîtres parmi des seigneurs plus puissants, et l'on vit peu à peu des entités toujours plus grandes se consolider, bien que des querelles de succession ou des soulèvements vinssent souvent remettre en cause les entités ainsi constitués. Norbert Elias a montré de quelle façon ces fluctuations dans un jeu de rivalités indépendantes ont pu faire place, dans une région donnée, à une situation dans laquelle une grande entité en était venue à dominer tous les autres belligérants, entité capable d'assurer la victoire de ceux d'entre eux qu'elle s'alliait. Dès lors, les petits seigneurs se livrèrent une concurrence pour obtenir les faveurs du puissant : on était entré dans le stade de la concurrence monopolistique, du « diviser pour régner». Dans une dernière phase, on vit ces petits souverains être mis un à un hors de combat en sorte que le plus puissant pût étendre son empire à toute la région : c'est à l'intérieur même du royaume ainsi créé que la compétition changea de nature. Elle devint une lutte politique, en principe pacifique, pour la conquête du pouvoir au sein des institutions de l'État ${ }^{26}$.

La société mondiale qui commence à se dessiner aujourd'hui n'est plus le théâtre de rivalités indépendantes et ne laisse pas non plus l'exercice du pouvoir dans les mains d'une seule autorité. Elle se situe dans une configuration que l'on peut imaginer entre ces deux extrêmes: les États ne se livrent pas une compétition ouverte, ils en sont réduits à s'assurer le soutien d'une « superpuissance ${ }^{27}$ ", c'est-à-dire jusqu'à une date récente les ÉtatsUnis ou l'Union soviétique, et aujourd'hui uniquement les États-Unis. Devenu le principal - pour ne pas dire l'unique - arbitre des conflits qui opposent les États entre eux, ce pays est à même de soutenir tel ou tel belligérant contre un autre au gré de ses propres intérêts. Les Nations Unies servent de couverture pour conférer à ces interventions une légitimité trans-

26. Norbert Euas, Het civilisatieproces, t. 2, 1939, 1969, Utrecht, Het Spectrum 1982, en part. chap. II, "Over de sociogenese van de staat ".

27. Pour un solide aperçu de la configuration mondiale des puissances, voir Paul KENNEDY, The Rise and fall of the great powers. Economic change and military conflict from 1500 to 2000, New York, Random House, 1987, et Theodore H. von LAuE, The World Revolution of westernization. The twentieth century in global perspective, New York/Oxford, Oxford University Press, 1987. 
nationale. Constater que la configuration mondiale actuelle est une forme à mi-chemin entre la compétition ouverte et l'exercice monopolistique du pouvoir ne doit pas nous amener à conclure automatiquement qu'on va aboutir à un monopole de violence, bien que ce scénario ne puisse pas non plus être exclu a priori.

Après un demi-siècle de guerre froide, les États-Unis apparaissent comme l'unique grande puissance. Aidés de leurs partenaires, ils semblent à même de pouvoir soumettre tout État et même toute coalition qui viendrait à s'opposer à eux. Il n'empêche que même avec le soutien de leurs alliés, ils ont été à plusieurs reprises incapables de restaurer l'ordre et la paix dans d'autres pays. S'ils semblent en mesure de vaincre un autre pays, ils ne parviennent pas à remplir les fonctions qu'assure un État à l'intérieur même de ses frontières. On songera par exemple aux limites de leur action contre l'Irak en 1991, alors même que l'État irakien était totalement défait par une coalition internationale. On songera aussi aux tentatives avortées de pacifier la Somalie, l'ex-Yougoslavie, ou encore le Rwanda ${ }^{28}$.

\section{COMMERCE MONDIAL ET SOCIÉTÉ TRANSNATIONALE}

Tout comme la configuration actuelle du pouvoir mondial, l'économie mondiale peut, elle aussi, être définie comme un état de concurrence monopolistique. Tandis que sur le plan politique, une configuration dominée par un acteur monopolistique s'est substituée à un duopole du pouvoir, on a vu, dans la sphère économique, deux économies planétaires laisser place à un unique système où règne un oligopole. Dans plus d'un secteur, quelques entreprises géantes commandent le commerce en contraignant leurs rivaux de taille plus modeste à adopter leurs normes et leurs prix. En règle générale, seules des multinationales de même niveau sont en mesure d'entretenir avec elles une véritable compétition. Ces concurrentes, le plus souvent constituées dans d'autres secteurs, affrontent sur la scène internationale leurs rivales déjà bien établies après avoir élargi leurs secteurs d'activités. On peut proposer une homologie entre l'analyse que propose Norbert Elias de la formation de l'État, selon le principe de l'accumulation et de la concentration des moyens coercitifs et celle de Karl Marx à propos de la compétition que se livrent les entrepreneurs capitalistes, envisagée comme un long processus d'accumulation et de concentration des moyens de production ${ }^{29}$.

28. Le cas haïtien, chasse gardée des États-Unis, mériterait une analyse des conditions dans lesquelles un semblant d'ordre légal a été établi après maintes tergiversations.

29. Voir N. EliAs, op. cit. supra n. 26, et K. MarX, Das Kapital, 1867, t. I, part. I, chap. xxIII, « Das allgemeine Gesetz der kapitalistischen Akkumulation ». 
Sur la longue durée, on peut observer un nombre toujours plus restreint d'acteurs commander une part toujours plus grande des moyens coercitifs et du capital économique.

Dans son étude consacrée à la formation de l'État en Europe, Charles Tilly s'est efforcé de réaliser une synthèse de ces deux lignes d'évolution en montrant que le processus de formation de l'État pouvait emprunter deux voies, soit principalement celle d'une accumulation intensive des moyens de production, soit celle d'une concentration intensive des moyens coercitifs. L'une et l'autre aboutissent à la structure relativement uniforme des États contemporains. Elle résulte des rapports de concurrence contraignants au sein du système global des États ${ }^{30}$. Bien que quelque peu schématique, l'exposé de Tilly nous fait avancer d'un grand pas dans la compréhension de la société transnationale actuelle, parce que justement les deux lignes du capital et de la contrainte s'y entremêlent - deux lignes que bon nombre d'auteurs tiennent à l'écart l'une de l'autre pour en privilégier une seule, cela au grand détriment d'une vision d'ensemble. Tout à fait malheureuse, cette dichotomie intellectuelle perdure. Ainsi, nombreux sont les conseillers économiques qui, appelés à assister les gouvernements des anciens pays communistes, ne tiennent aucun compte des fonctions vitales que remplit l'État dans la formation et le fonctionnement des marchés ${ }^{31}$. Se focaliser de la sorte sur la vie économique et rester aveugle à tout ce qui concerne la tâche de l'État s'avère dès lors très préjudiciable à ces sociétés.

On peut définir la société transnationale comme une configuration de relations de concurrence liée ou oligopolistique, tant au sens économique qu'au sens politique. Mais il est, d'autre part, possible de la caractériser en tant que série de relations concentriques et hiérarchiques : comme une configuration centre/périphérie de domination et d'exploitation, au sens avancé par Immanuel Wallerstein. Les auteurs que l'on range dans son école du système mondial s'accordent pour s'intéresser exclusivement à l'expansion planétaire de l'économie capitaliste, soutenant, dans la droite ligne de la tradition néo-marxiste, que toute domination militaire et politique découle d'un impérialisme mu par des forces économiques.

Malgré les limites qui sont les siennes, cette approche en termes de centre et de périphérie livre une première caractérisation globale dont on ne

30. Voir Charles Tilly, Coercion, capital and European states, Cambridge, MA/Oxford, Blackwell, 1990.

31. Au sujet des conseils donnés à ces gouvernements par les organisations internationales, voir Bob DeAcon, "Global Social Policy Actors and the shaping of post-communist social policy ", in Social Policy beyond borders. The social question in transnational perspective, éd. Abram de Swaan, Amsterdam, Amsterdam University Press, 1994; et Bob Deacon, Michelle HuLSE, "The Council of Europe, the European Union, the OECD, the World Bank and the shaping of East-European social policy. A case of varying advice and varying intervention », texte d'une conférence donnée lors du "Third Prague international workshop on social responses to transformations in East-Central Europe ", Prague, du 6 au 8 mai 1994. 
saurait se passer. Il s'agit, qui plus est, d'une généralisation adéquate pour étudier les modèles mondiaux d'échanges et de domination culturels. Cela n'est pas tellement surprenant étant donné que les échanges culturels suivent pour une grande part les voies tracées durant la période de l'expansion coloniale de l'Europe. Il en va de même de la structure de l'hégémonie politique post-coloniale et des relations commerciales capitalistes.

En effet, on observe d'abord ce modèle concentrique ou hiérarchique des échanges culturels dans l'exploitation manifestement commerciale que l'on fait de la culture populaire pour toucher un très large public: films, musique pop et shows télévisés, tous diffusés par le moyen des réseaux planétaires des médias de masse modernes. On l'observe également dans les flux des articles de marque qui revêtent de telles connotations symboliques que leur valeur symbolique, justement, en arrive à dépasser leur valeur d'usage. On a encore démontré récemment que la diffusion mondiale et la «popularité différentielle» des sports modernes suivent des modèles de rapports de classes parmi les nations et, dès lors, obéissent à la configuration mondiale de l'hégémonie politique ${ }^{32}$. À cela il convient d'ajouter que les touristes eux aussi empruntent des routes qui ont été tracées par les soldats, les marchands, les missionnaires et les administrateurs, des routes pavées depuis lors par les entreprises transnationales.

SYSTÈME MONDIAL DES LANGUES ET SOCIÉTÉ TRANSNATIONALE

On retrouve des modèles analogues pour ce qui est de l'expansion des grandes langues, en premier lieu au sein des nations mêmes, puis sur toute la surface du globe. La sociologie politique des langues ${ }^{33}$ définit ces lignes de rivalité et d'accommodement entre groupes de langues en recourant à une « configuration florale » qui correspond au modèle centre/périphérie : au cours du processus de formation des États et des nations, un idiome se

32. Voir Maarten VAN BotTEnBURG, Verborgen competitie. Over de uiteenlopende populariteit van sporten, Amsterdam, Bert Bakker, 1994; Ruud STokvis, "The international and national expansion of sports ", in Sports in Asia and Africa, éd. Eric A. WAGNER, New York, Greenwood Press, 1989, p. 13-24.

33. Voir Language Spread. Studies in diffusion and social change, éd. Robert L. COOPER, Bloomington, IN, Indiana University Press, 1982; The Other Tongue. English across cultures, éd. Braj B. KACHRU, Urbana/Chicago/Londres, University of Urbana Press, 1982; David D. Laitin, Language Repertoires and State construction in Africa, Cambridge, Cambridge University Press, 1992; Ali A. Mazrui, The Political Sociology of the English language. An African perspective, Paris/La Haye, Mouton, 1975; " The emergent world language system ", no spéc. éd. par A. DE SWAAN, International Political Science review, vol. 14, 3, juil. 1993. 
dégage de l'ensemble des langues et des dialectes pour devenir la " langue centrale " parlée par - presque - tous ceux qui possèdent plus d'une langue; cet idiome en tire une position stratégique particulière pour ce qui relève des communications interrégionales et nationales ${ }^{34}$. L'histoire montre qu'il s'est agi à chaque fois de la langue parlée à la cour et dans la capitale, identique le plus souvent à celle du capital économique. L'extension massive de l'enseignement primaire a permis à cet idiome de devenir de surcroît le véhicule de l'enseignement sur tout un territoire; durant le processus de formation de l'État, il s'est peu à peu imposé comme langue universelle de la nation, parlée par presque tous les habitants avant de devenir l'unique langue couramment utilisée dans le pays. C'est en France qu'un tel processus d'unification linguistique a été poussé le plus loin. S'il y en eut de semblables aux Pays-Bas, en Scandinavie, en Italie, en Allemagne, en Russie, au Japon et - dans un contexte d'immigration massive - aux États-Unis, en Australie, au Brésil et dans les autres pays d'Amérique latine, on relèvera plusieurs cas connus pour lesquels de tels processus, à l'œuvre certes, ne sont pas encore achevés. Telles sont l'Angleterre, l'Espagne et la Chine.

Dans les anciennes colonies, divers idiomes indigènes d'importance s'affrontèrent pour la suprématie et rivalisèrent, en outre, avec la langue importée par les colonisateurs, la seule au bout du compte qui rapprochait les élites nationales des différents peuples de l'empire et qui opérait comme la langue centrale. Des rapports de «jalousie de groupe à groupe» ont empêché que s'impose alors une langue locale alors même que la revendication d'identité nationale excluait d'accepter l'ancienne langue coloniale comme moyen de communication pour l'ensemble du pays ${ }^{35}$. C'est le principe du double dilemme propre à des pays comme le Nigeria, l'Inde, le Ghana ou le Zaïre : l'ancienne langue coloniale devait-elle devenir le véhicule de la communication nationale ou bien fallait-il finalement opter pour une langue indigène? Et dans ce dernier cas, quelle langue retenir? Dans la pratique, cette impasse a favorisé la généralisation de la langue héritée des colonisateurs - principalement l'anglais et le français —, celle que les enfants choisirent d'apprendre à l'école comme deuxième langue pour élargir leurs perspectives d'avenir et pour mieux profiter des divertissements de masse.

L'unification linguistique dans les vieilles nations et la coexistence d'anciennes langues transmises par les colonisateurs avec des langues indi-

34. Voir A. DE SwaAN, "Het lager onderwijs als code voor nationale communicatie ", in Zorg en de staat. Welzijn, onderwijs et gezondheidszorg in Europa et de Verenigde Staten in de nieuwe tijd, Amsterdam, Bert Bakker, 1989.

35. Voir D. LaItin, op. cit. supra n. 33; A. DE SwaAN, «Language politics in India and Europe. A comparison based on a model of conflict of language interests ", Journal of educa. tional and social change, (Pune), 5-3 oct.-déc. 1991, p. 13-34. 
gènes dans les jeunes États ont contribué à la formation d'un système mondial de langues relativement cohérent dont l'unité est garantie par ceux qui, possédant plus d'une langue, assurent la communication entre les différents groupes linguistiques. Dans cette configuration mondiale des langues, les idiomes nationaux opèrent chacun comme langue centrale face à des dialectes régionaux et ethniques, telles des planètes autour de chacune desquelles gravitent autant de lunes. À un niveau au-dessus, on recense quelques langues qui occupent une position qu'on peut qualifier de «supercentrale » relativement à une série d'autres langues nationales et centrales, un peu à l'image du soleil entouré de ses planètes. Le russe, le chinois, l'arabe, l'hindi, l'indonésien (le malais), l'espagnol, le français et, bien entendu, l'anglais sont toutes des langues parlées par au moins cent millions de personnes, chacune réunissant en un halo une série de langues elles-mêmes centrales mais à une échelle seulement nationale. Si un nombre comparable de locuteurs parlent le portugais, l'allemand et le japonais, il manque à ces trois langues la caractéristique de fonctionner à la manière de maillons interlinguistiques; à l'inverse, le swahili, idiome parlé par un nombre plus petit de personnes, opère comme un moyen de communication transnationale. Ensemble, ces douze langues donnent un accès direct à environ soixante pour cent de la population mondiale. Enfin, il est une langue qui, située au cœur de ce système galactique et entourée d'une douzaine de systèmes solaires, occupe une position centrale entre toutes ces langues supercentrales : il s'agit de l'anglais, le véhicule qui relie entre eux les locuteurs de toutes les autres langues mondiales.

Cette métaphore astronomique des différences de niveaux entre voie lactée, systèmes solaires, planètes et lunes est bien entendu une reproduction verbale d'un diagramme concentrique de Venn ou d'un modèle hiérarchique, un développement du modèle primaire centre/périphérie. Tout simplifié qu'il soit, il nous montre l'ordre rigoureux qui règne au cœur d'un embrouillamini de quelque chose comme cinq mille langues parlées sur la planète. Un système mondial de langues est en voie de formation.

L'ordre rigoureux que renferme cette image planétaire des langues peut nous servir par ailleurs de modèle pour analyser d'autres phénomènes d'échanges culturels qui, eux aussi, se caractérisent par la dynamique de la concurrence oligopolistique et par le modèle hiérarchique d'un centre cerné de cercles concentriques de périphéries, elles-mêmes « semi-périphéries " fonctionnant comme centres seconds pour des périphéries d'un échelon inférieur.

L'examen de la société transnationale met en évidence un ordre et une dynamique tout à fait singuliers, une autonomie relative non seulement au regard des réseaux des sociétés nationales et des marchés transnationaux dont elle est constituée mais également par rapport aux participants : États, 
entreprises et organisations transnationales. La structuration de la société transnationale se déroule selon un processus de compétition oligopolistique, d'élimination, de standardisation et d'homogénéisation des éléments qui subsistent. Et la même chose vaut, moyennant spécification, pour la mondialisation de la culture.

LE SYSTĖME CULTUREL MONDIAL

Qu'il s'agisse du sport, du tourisme, des divertissements de masse, de la musique pop, de la danse, de la mode ou des vêtements de marque, on retrouve à chaque fois le même modèle d'une multiplication - déconcertante et toujours plus rapide - des biens et des services offerts aux quatre coins du monde moderne : «On trouve tout en principe partout, mais c'est l'environnement qui sélectionne ${ }^{36}$. » La généralisation au niveau planétaire de tels produits et services entraîne l'abandon de certaines traditions locales, mais il arrive souvent aussi qu'elles demeurent en subissant une transformation et en se retrouvant mêlées à des styles et des produits nouveaux provenant de pays lointains. C'est ce qu'Ulf Hannerz a qualifié de « créolisation », la métaphore linguistique renvoyant autant à l'abâtardissement culturel qu'à la créativité propre au niveau local. Régulièrement, on réexporte ces produits indigènes " croisés » en métropole d'où ils repartent par la voie du réseau mondial jusqu'aux endroits les plus reculés de la planète. Dans ce domaine aussi, la musique nous offre un cas emblématique. Après que la musique pop afro-américaine eut débarqué en Jamaïque, les Jamaïcains firent en retour cadeau au monde du reggae. Le reggae gagna ensuite l'Afrique d'où le jùjú et la world music partirent conquérir le monde ${ }^{37}$. L'alimentation livre un second exemple: les recettes conçues dans le monde occidental qui sont la base de la restauration rapide ont été imitées par des immigrés du Moyen-Orient qui inventèrent des versions adaptées à leur propre cuisine : le falafel ou le gyro. Les immigrés indiens transformèrent leurs plats au curry. Les Mexicains firent de même de leurs tacos et enchiladas. Et tous ces produits d'importation sont maintenant devenus des composantes à part entière de la restauration rapide de nos grandes villes. Tout compte fait, on assiste partout au niveau local à un

36. Cet adage est connu dans la botanique comme la « loi de Beyerinck» sur la dissémination des espèces végétales.

37. Voir Christopher A. WATERMAN, Jùjú. A social history and ethnography of an African popular music, Chicago, University of Chicago Press, 1990; et Peter ManUEL, Popular Musics of the non-Western world, New York, Oxford University Press, 1988. 
accroissement de la variété des produits, une offre qui ne cesse en même temps de présenter, dans chaque endroit donné, de plus en plus de similitudes avec celle de n'importe quel autre lieu. De telles images ruinent les conclusions simplistes décrivant une mondialisation de la culture sous l'empire d'une seule norme, et dans laquelle l'Occident détruirait les formes de cultures locales en imposant à tous les peuples ses propres plats standardisés.

Il est ainsi possible de définir la mondialisation comme un processus par lequel, dans un nombre croissant d'endroits, la variété de l'offre s'accroît finalement, en même temps que cette offre plus diversifiée présente pour chaque endroit donné toujours plus de similitudes avec celle de tous les autres lieux. En d'autres mots : la mondialisation procède d'une hétérogénéisation locale qui va de pair avec une homogénéisation globale.

AMENDEMENTS À APPORTER AU MODÈLE HIÉRARCHIQUE

Retenir un modèle centre/(semi-)périphérie de compétition oligopolistique est la manière la plus adéquate d'approcher la société transnationale actuellement en devenir. Mais il convient d'apporter tout de suite des amendements à ce premier et double schéma, et ce sur cinq points.

Tout d'abord, il existe des centres et des périphéries qui varient selon les domaines de la société transnationale. Le Japon, par exemple, pays qui a connu partout un succès exceptionnel dans le lancement d'articles électroniques de marque, n'a pratiquement influencé en aucune façon la culture populaire transnationale en d'autres domaines (pas plus d'ailleurs que la culture élitaire).

Deuxièmement, même pour un domaine particulier, il n'est pas nécessaire qu'il existe un réseau uniforme formé autour d'un centre, ou même que ce centre se transforme progressivement en une semi-périphérie, puis une périphérie. Ainsi, par exemple, si la musique populaire se répand à partir d'un centre américain, il s'avère qu'une partie importante de l'Asie n'en laisse pratiquement rien passer - il en va de même pour les films américains - orientée qu'elle est sur l'industrie culturelle populaire de Bombay. Apparemment, plusieurs centres peuvent coexister pour couvrir un seul domaine, tous transnationaux sans qu'aucun ne soit vraiment planétaire.

En troisième lieu, entre le centre et la périphérie, les choses ne se passent pas obligatoirement dans un seul sens : il existe un contre-courant constant de biens de consommation, de musique, de danse, de sport, de littérature, de cuisine, de vêtements, de décoration, etc., qui part de la zone péri- 
phérique pour revenir au centre, avant de repartir vers d'autres régions périphériques. Relevons, toutefois, que ces mouvements suivent des réseaux orientés depuis les centres de l'industrie musicale, de l'édition, du monde de la mode ou de la publicité, etc., en subissant une adaptation aux langues et aux codes culturels (aux cadres, aux frames, pour utiliser le terme d'Erving Goffman) du centre et en y étant intégrés ${ }^{38}$.

En quatrième lieu, ces courants planétaires de culture populaire et commerciale ne balayent pas nécessairement les expressions artistiques, musicales, vestimentaires et coutumières traditionnelles d'une région donnée. C'est un fait, les anciens genres viennent parfois à disparaître. Une culture de masse étrangère peut en être la cause mais aussi l'abandon des formes de vie qui lui correspondent, effet de l'urbanisation et de l'industrialisation. Mais il arrive tout aussi souvent que les genres qui subsistent soient croisés avec des styles étrangers, donnant naissance à ce que les musiciens appellent des crossover et que les autochtones conservateurs qualifient avec mépris de «bâtardisation ».

En cinquième lieu enfin, il est tout à fait impossible de distinguer les personnes selon qu'elles seraient « locales » ou " cosmopolites » (pour ne pas dire « transnationales »). En effet, à mesure que l'urbanisation s'intensifie et que les médias de masse pénétrent plus avant les campagnes, chacun peut plus souvent et plus facilement adopter un registre ou bien l'autre, oscillant en quelque sorte d'un penchant à l'autre, local, national ou transnational, tout comme chacun peut passer d'un code de langue à un autre (ou, pour employer l'expression anglaise spécialisée, jouer sur le code switching) : dialecte régional, idiome national, langue mondiale. Ces passages s'opèrent en général de manière spontanée et sans que les intéressés euxmêmes y songent, étant entendu que cela peut aussi se produire en toute conscience et donner lieu à de vifs conflits. On touche ici précisément ces pratiques de passage, inscrites dans des contextes locaux et dans l'environnement immédiat, qui constituent l'objet de la recherche microsociologique des pratiques transnationales in situ.

Jusqu'ici, il a été question des processus qui gouvernent la mondialisation sans qu'il ne soit véritablement fait référence aux personnes qu'on considérerait comme des acteurs de ces processus. Ces personnes furent envisagées comme des consommateurs, des employés, des auditeurs ou encore des spectateurs anonymes et relativement passifs. Mais les réseaux mondiaux sont mis en place par des gens énergiques et entreprenants tandis que des entreprises et des institutions considérables en assurent la coordination. La sociologie de la société transnationale doit donc enquêter empi-

38. Erving GoffMAn, Frame Analysis. An essay on the organization of experience, Cambridge, MA, Harvard University Press, 1974. 
riquement sur ces entrepreneurs, ces fonctionnaires, ces experts et ces célébrités médiatiques qui incarnent ces réseaux. Il s'agit notamment de scruter les points de rencontre entre les réseaux nationaux - par exemple, des fonctionnaires et des experts d'un pays donné - et internationaux - par exemple, des dirigeants et spécialistes qui opèrent au niveau international. Ainsi, un groupe relativement restreint d'experts internationaux, qui travaillent au sein d'institutions occupant des positions stratégiques et qui collaborent avec des fonctionnaires de différents pays, peut jouer un rôle capital dans la généralisation planétaire de l'enseignement, de la législation du travail, du secteur de la santé, de la réglementation fiscale, etc. De même, ce sont de petits groupes de planificateurs urbains et d'architectes qui ont établi les normes ayant effectivement servi à planifier et à aménager les villes. Plus ou moins protégés par les gouvernements nationaux, les magnats des médias sont, de leur côté, parvenus à mettre en place des réseaux transnationaux pour la radio et la télévision par satellite. En bref, il s'avère que des personnes bien informées, qui interviennent au moment propice et se montrent convaincantes, peuvent pénétrer les réseaux mondiaux avec une facilité surprenante.

L'approche sociologique de la société transnationale telle qu'on la présente dans ces pages nous a permis d'éviter de tomber dans un des trois pièges suivants. Le premier risque était d'extrapoler et d'hypertrophier tout bonnement des concepts sociologiques dont le sens a été arrêté pour la société nationale en vue de les transformer en un répertoire propre à la société transnationale. Le deuxième était de répéter des recherches déjà accomplies dans d'autres disciplines comme les relations internationales, l'économie, les sciences politiques, le droit. Enfin, on risquait de se limiter un peu trop rapidement aux seuls aspects strictement culturels. Les sociologues auront mieux à faire en prenant pour objet la société transnationale dans son ensemble, croisant leurs approches avec d'autres branches des sciences sociales et, cela fait, en délimitant à l'intérieur de ce cadre un objet de recherche propre à la sociologie: le modèle des rapports qui relient les personnes entre elles par-delà des frontières et, sur ce fond, les institutions qui servent d'instrument lors de la formation de tels réseaux.

MICROSOCIOLOGIE ET MACROSOCIOLOGIE DE LA SOCIÉTÉ TRANSNATIONALE

Il est à espérer que la recherche s'oriente sur les relations transnationales entre individus, que ces relations s'établissent directement ou indirectement, par le biais d'organisations, d'entreprises transnationales ou d'États. De telles relations ne représentent qu'une part du réseau propre à un indi- 
vidu, certes, mais bien la part qu'il s'agit de considérer quand on s'intéresse à ces "pratiques de passage " qui lui permettent de jouer d'un registre à un autre, d'une inflexion locale à une inflexion nationale, et de celle-ci à une autre mondiale ou inversement, tout cela le plus souvent dans le court intervalle de temps d'une conversation téléphonique ou lors d'une rencontre fortuite.

La façon dont de tels passages instantanés sont " mis en scène » est un indice de la réalité de la société transnationale en même temps qu'un moment de sa réalisation. Bien que la société mondiale n'existe pas en un endroit précis et unique, il n'en est pas moins possible de l'étudier in situ, c'est-à-dire partout où des gens se retrouvent dans une situation qui leur donne la possibilité de passer d'un niveau d'orientation à un autre.

Un autre site, tout à fait stratégique pour l'étude de la société transnationale est l'étude des agences transnationales, ces institutions composées d'un personnel international et fonctionnant grâce à un réseau de rapports avec des experts et des fonctionnaires de gouvernements nationaux. De tels réseaux développent souvent une dynamique propre dans laquelle de hauts fonctionnaires d'un État peuvent occuper une position qui dépasse assez largement les intérêts nationaux qu'ils sont chargés de défendre ${ }^{39}$. Ce mécanisme est loin d'être étranger à la diffusion d'un ethos de la santé préventive, de l'enseignement de masse, de la protection de la nature et des droits des citoyens, y compris au sein des puissances périphériques apparues à une époque plus récente dans le système étatique mondial.

Les métaphores de la compétition oligopolistique et des relations centre/ (semi-)périphérie ressemblent beaucoup, d'un point de vue formel, au schéma de la «configuration florale » d'une langue centrale liée à des langues régionales et des dialectes, grâce à une élite composée de personnes bilingues qui toutes possèdent la première langue dans leur répertoire. Aussi, l'étude du système mondial des langues en voie de formation aujourd'hui est-il utile à plus d'un titre en vue d'une première exploration de la société transnationale prise dans son ensemble.

Une image d'ensemble de la société mondiale en devenir peut nous apparaître si on analyse des statistiques mondiales, lesquelles se laissent lire à deux niveaux. Premièrement, en tant que données qui nous fournissent des informations sur les sociétés nationales actuelles (par exemple, des chiffres servant à comparer les chiffres du produit national brut, le pourcentage d'enfants scolarisés, l'espérance de vie moyenne, la fécondité

39. Voir au sujet de l'environnement la contribution d'August GuswiJt à Nico Wilterdink et Bart Van HeErikHuzen, Mondialisering. De wording van de wereldsamenleving, rééd, Groningue, Wolters-Noordhoff, 1995; pour l'enseignement, John W. MEYER et al., School knowledge for the masses. World models and national primary curricular categories in the twentieth century, Washington, DC, Falmer Press, 1992. 
conjugale...) et sur les échanges internationaux (par exemple, le commerce, le tourisme, les communications téléphoniques) ${ }^{40}$. Mais on peut, en second lieu, étudier ces statistiques comme des éléments qui eux-mêmes contribuent à la culture transnationale qu'on leur demande de cerner : elles contiennent, en effet, les concepts clés et les données de base qui permettent une comparaison sur le plan international, comparaison qui suscite la rivalité entre gouvernements nationaux pour faire plus (ou, en tout cas, pas beaucoup moins) que les autres. De la sorte, ces statistiques contribuent à la réalisation d'objectifs de développement que ces mesures quantitatives contiennent de manière implicite, voire même explicite.

Pour autant que la sociologie vise à accomplir sa tâche originaire de science globale de la société humaine, il faudra bien briser les frontières tracées entre la recherche nationale et la recherche régionale et, dès lors, assortir les études comparées d'analyses de la configuration mondiale considérée dans sa totalité. C'est, pour la sociologie, l'unique façon d'affronter la réalité d'une société mondiale en formation. Ce programme commence simplement par l'étude des relations transnationales, liens de plus en plus denses aujourd'hui qui nouent entre tous les êtres humains un réseau mondial, concret et vaste.

Abram DE SwaAN (janvier 1998) ${ }^{41}$.

40. Voir Alain DesRosières, La Politique des grands nombres. Histoire de la raison statistique, Paris, La Découverte, 1993.

41. Cet article a été traduit par Daniel Cunin, en collaboration avec Matthijs Engelberts, grâce à une subvention de la Koninklijke Nederlandse Academie van Wetenschappen (KNAW). La traduction a été révisée par l'auteur et la rédaction. 